\title{
Point-of-care bedside ultrasound examination for the exclusion of clinically significant ankle and fifth metatarsal bone fractures; a single blinded prospective diagnostic cohort study
}

\author{
Aniek Crombach ${ }^{1 *}$ (D, Nasim Azizi ${ }^{1}$, Heleen Lameijer ${ }^{2}$, Mostafa El Moumni ${ }^{3}$ and Jan C. ter Maaten ${ }^{4}$
}

\begin{abstract}
Objective: The aim of this study was to assess the diagnostic value of point-of-care bedside ultrasound (PoCUS) as in usual clinical practice in suspected ankle and fifth metatarsal bone fractures, compared to the standard of radiographic imaging.

Methods: This prospective study included patients $\geq 17$ years presenting to the Emergency Department with ankle trauma and positive Ottawa Ankle Rules. All patients underwent PoCUS of the ankle by a (resident) emergency physician, the images were assessed by an ultrasound expert. Both were blinded for the medical history and clinical findings of the patients. Radiography of the ankle followed, evaluated by a radiologist blinded from the PoCUS findings. Primary outcome measures were sensitivity and specificity of PoCUS.

Results: A total of 242 patients were included, with 35 (22\%) clinically significant (non-avulsion) fractures observed with radiography. The sensitivity of PoCUS in detecting clinically significant fractures by all sonographers was $80.0 \%$ (95\% Confidence Interval (Cl) 63.0 to 91.6\%), specificity 90.3\% (95\% Cl 83.7 to 94.9\%), positive predictive value $70.0 \%$ (95\% Cl 57.0 to $80.3 \%$ ) and the negative predictive value $94.1 \%$ (95\% Cl 89.1 to $96.9 \%$ ). The sensitivity of PoCUS in detecting clinically significant fractures by the expert was $82.8 \%(95 \% \mathrm{Cl} 66.3$ to $93.4 \%)$, specificity $99.2 \%$ (95\% Cl 95.5 to $99.9 \%$ ), positive predictive value $96.7 \%$ (95\% Cl 80.3 to $99.5 \%$ ) and the negative predictive value $95.3 \%$ (95\% Cl 91.0 to $98.2 \%)$.
\end{abstract}

Conclusion: PoCUS combined with the OAR has a good diagnostic value in usual clinical practice in the assessment of suspected ankle and fifth metatarsal bone fractures compared to radiographic imaging. More experience with PoCUS will improve the diagnostic value.

Trial registration: Registered in the local Research Register, study number 201500597.

Keywords: Point-of-care bedside ultrasound, PoCUS, Emergency ultrasound, Bedside ultrasound, Emergency department, Ankle fractures, Foot fractures

\footnotetext{
* Correspondence: aniekcrombach@hotmail.com

'Department of Emergency Medicine, University Medical Centre Groningen, University of Groningen, Hanzeplein 1, 9700 RB Groningen, the Netherlands Full list of author information is available at the end of the article
}

(c) The Author(s). 2020 Open Access This article is licensed under a Creative Commons Attribution 4.0 International License, which permits use, sharing, adaptation, distribution and reproduction in any medium or format, as long as you give appropriate credit to the original author(s) and the source, provide a link to the Creative Commons licence, and indicate if changes were made. The images or other third party material in this article are included in the article's Creative Commons licence, unless indicated otherwise in a credit line to the material. If material is not included in the article's Creative Commons licence and your intended use is not permitted by statutory regulation or exceeds the permitted use, you will need to obtain permission directly from the copyright holder. To view a copy of this licence, visit http://creativecommons.org/licenses/by/4.0/. The Creative Commons Public Domain Dedication waiver (http://creativecommons.org/publicdomain/zero/1.0/) applies to the data made available in this article, unless otherwise stated in a credit line to the data. 


\section{Background}

Foot and ankle injuries are one of the most frequent reasons to visit the Emergency Department (ED), but the diagnostic instruments used are highly inefficient [1]. Plain radiographs are commonly used to diagnose a suspected fracture in foot and ankle trauma. The relative low probability of fractures causes frequent unnecessary radiation exposure, together with burdening of the patient and costs, time, and crowding in the ED. [2]

To reduce the use of radiography for the assessment of ankle injuries the Ottawa Ankle Rules (OAR) were developed [2-4]. The OAR has a high sensitivity but are low in specificity, leading again to a lesser but still significant amount of unnecessary radiation exposure in these patients $[2,4]$.

A few studies have assessed the use of point-of-care bedside ultrasound (PoCUS) in diagnostics of foot and ankle injuries specifically, showing a sensitivity ranging from 87.3 to $100 \%$ and specificity ranging from 90.1 to 99.1\%. These studies show that when PoCUS is used in OAR positive patients, there can be an approximately $80 \%$ reduction of radiological assessment. The studies on the matter are all subjective to bias regarding PoCUS, because of unblinded designs, limited amount of and selected sonographers and non-consecutive inclusion [2, 5].

In this prospective single blinded cohort study we aim to assess the diagnostic value of PoCUS in suspected fifth metatarsal bone and ankle fractures compared to the use of radiographic imaging.

\section{Methods \\ Study desig}

This was a single blinded prospective diagnostic cohort study, conducted between August 2015 and December 2017. The goal was to compare PoCUS to the reference standard of radiographic imaging. Approval by the local ethics committee was obtained and patients informed consent documented. The Standards for Reporting Diagnostic Accuracy (STARD) statement checklist was used for reporting [6].

\section{Study setting and population}

This study was conducted in the ED of the University Medical Centre in Groningen, the Netherlands, a tertiary trauma centre receiving around 900 patients with a foot or ankle trauma per year. Patients presenting to the ED with a foot or ankle trauma were assessed according to standard clinical protocol. The triage nurses were trained to apply and document the OAR (Table 1). If positive, the patients were screened for eligibility [7].
Table 1 Ottawa Ankle Rules [3].

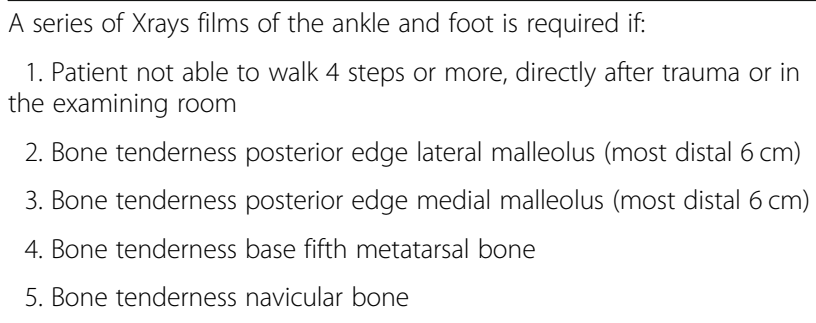

\section{Inclusion and exclusion criteria}

Inclusion criteria were age $\geq 17$ years, inversion or eversion trauma mechanism and a positive OAR. Exclusion criteria were negative OAR, open fractures, visible major dislocation, multi-trauma patients, previous fracture of the same ankle, degenerative ankle disease and a trauma $\geq 48 \mathrm{~h}$, no informed consent, other fractures (calcaneus, tertiary malleolus), no blinding for the specific outcome of the OAR and the trauma mechanism, included after the closing date of the study [1, 5, 8-10]. Patients of whom the records were not evaluable were ineligible, avulsion fractures were excluded. (Fig. 1).

There was inclusion of consecutive patients, done by all the emergency physicians (EP's) or residents, as in usual clinical practice. Informed consent and PoCUS were performed by an EP or resident. The sonographer was blinded for the trauma mechanism and the specific outcome of the OAR, by not knowing specifically which rules of the OAR led to a positive finding. The patient would consecutively be assessed and treated, in accordance with current practice, by another ED doctor blinded for PoCUS outcome. Radiography of the ankle included anteroposterior and lateral radiographs of the ankle, and fifth metatarsal bone if appropriate.

\section{Sonographers}

All of the sonographers were either EP's or residents with experience in PoCUS, trained with standardized sonography courses in emergency medicine. In total 23 sonographers were part of this study. These were 11 EP's, 12 residents, and 1 EP expert sonographer. The expert sonographer was trained within the Ultrasound Leadership Academy Fellowship, a 12-month comprehensive course of PoCUS [11]. All sonographers were additionally trained by the expert in a 2-h theoretical and practical training specifically for PoCUS of the fifth metatarsal bone, distal tibia and fibula.

\section{Study protocol}

PoCUS was performed initially on a Zonare Z One ultrasound machine, but after mechanical failure a SonoSite $\mathrm{X}$-porte was used. A $10-\mathrm{MHz}$ linear probe was used for sonography of 3 regions of the ankle: 


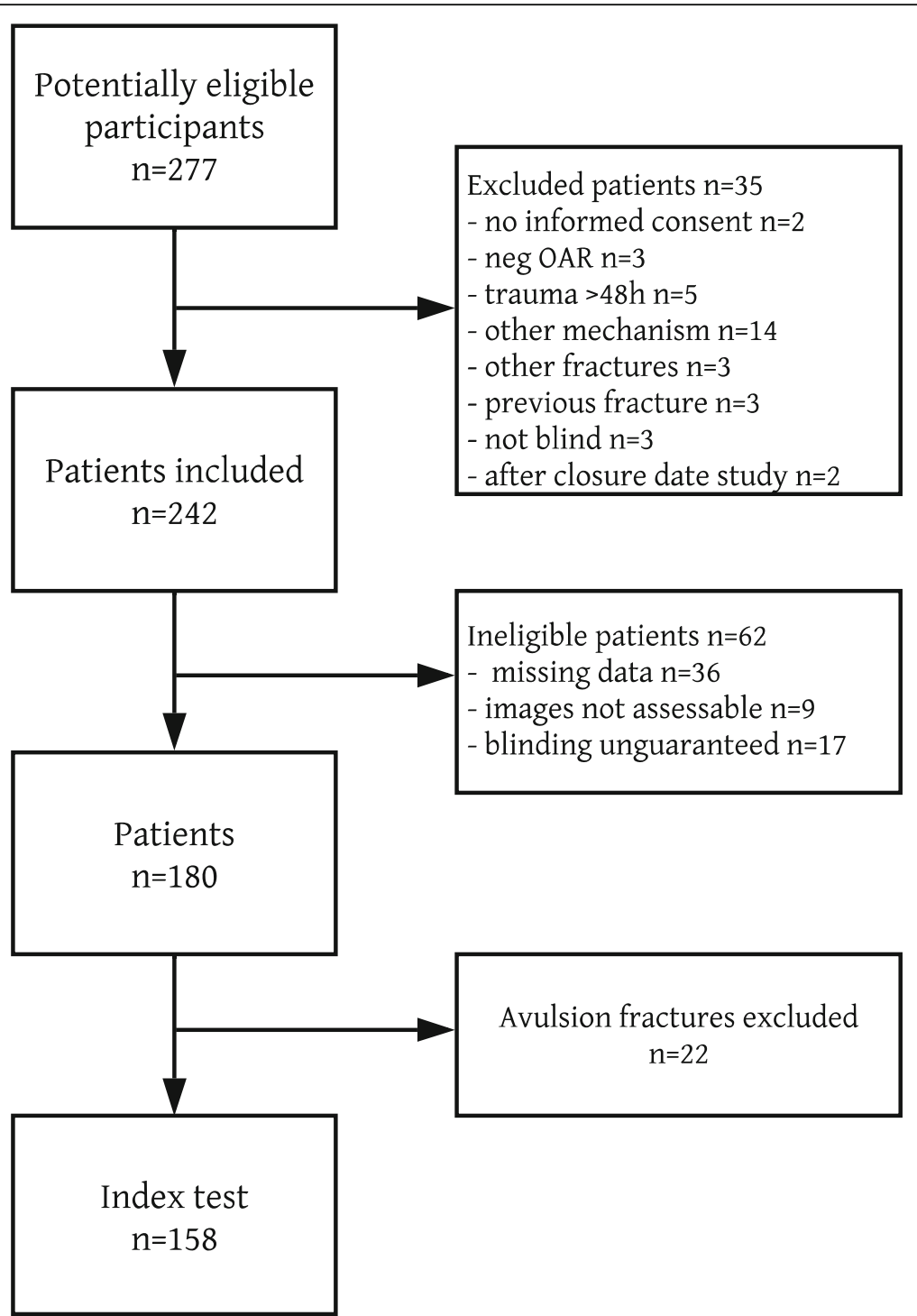

Fig. 1 The process of inclusion, with the eligible, excluded and ineligible patients and avulsion fractures

1. Up to $10 \mathrm{~cm}$ proximal of the distal tibia.

2. Up to $10 \mathrm{~cm}$ proximal of the distal fibula.

3. Up to $5 \mathrm{~cm}$ proximal of the distal fifth metatarsal bone.

PoCUS was focussed on cortical disruption, indicating a fracture. PoCUS was not used to identify soft tissue or syndesmotic injuries of the ankle. The navicular bone was not scanned in this study with regards to low diagnostic value in previous research [10]. A 10-s sonography video of all patients was recorded to visualize the area of interest. Criteria for diagnosis of a fracture with PoCUS were cortical disruption or axial deviation of the bone surface as observed by the sonographer. See Fig. 2 for an example of a cortical disruption as seen by PoCUS. Fractures $<3 \mathrm{~mm}$ in width were considered

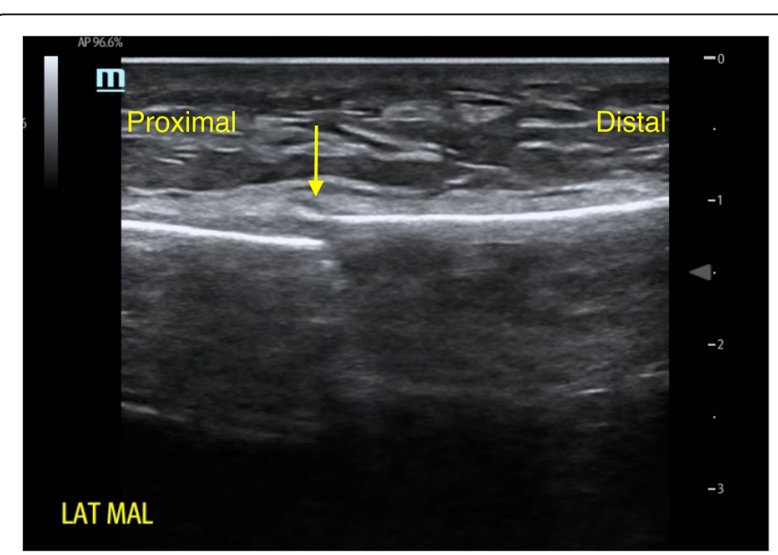

Fig. 2 Ultrasound image of the lateral malleolus (= lat mal). The arrow points at cortical disruption 
non-significant avulsion fractures, as per previous studies [5].

All videos were secondarily assessed by the expert. Any differences between sonographers and expert sonographer were documented. The final evaluation of the radiographic images by the radiologist was considered the golden standard for the diagnosis of a fracture. The radiologist was blinded for the outcome of the PoCUS, but not for clinical findings that were documented. The result of the reference standard was not available to the sonographers or expert.

\section{Data analysis and sample size calculation}

A sample size was calculated, using criteria based on previous research and desired study characteristics; a prevalence of patients with a fracture of $25 \%$, a minimal acceptable sensitivity of $96 \%$ and minimal acceptable specificity of $97 \%$, with a confidence interval of $5 \%$ for sensitivity. The calculated sample size for the determined sensitivity was 236 and for the specificity 62 patients [12].

Data analysis was done in SPSS Statistics 23 (IBM, Armonk, New York, USA). All demographics and clinical data were imported. Comparison between categorical variables was calculated by the chi-square test.

The measures of diagnostic accuracy were sensitivity and specificity of PoCUS for detection of fractures of the lateral and medial malleoli and fifth metatarsal bone compared to the golden standard radiographic images. Distal avulsion fractures of the fibula were not considered as a clinically relevant fracture, but mentioned [1].

\section{Results}

A total of 277 patients could be assessed for eligibility. In total 242 were included, of which 62 ineligibles. The records of these ineligible patients were not evaluable due to missing data, non-assessable videos or because blinding was not guaranteed (thus when patients were included by the expert sonographer). Of the remaining 180 patients, 22 patients with avulsion fractures were excluded, leaving 158 patients for analysis, see Fig. 1. Baseline criteria and results are presented in Tables 2 and 3.

In 35 patients $(22 \%)$ significant fractures were seen using radiography. The sonographers and the expert identified 28 and 29 fractures, and missed 7 and 6 fractures, respectively. (see Tables 3, 4).

The sensitivity of PoCUS in detecting clinically significant fractures by all sonographers was $80.0 \%$ (95\% Confidence Interval (CI) 63.0 to $91.6 \%$ ), specificity $90.3 \%$ (95\% CI 83.7 to $94.9 \%$ ), positive predictive value $70.0 \%$ (95\% CI 57.0 to $80.3 \%$ ) and the negative predictive value 94.1\% (95\% CI 89.1 to $96.9 \%$ ). The sensitivity of PoCUS in detecting clinically significant fractures by the expert was $82.8 \%$ (95\% CI 66.3 to $93.4 \%$ ), specificity $99.2 \%$ (95\%

Table 2 Baseline criteria, including non-significant fractures

\begin{tabular}{lll}
\hline & Patients (n) & Percentage \\
\hline Total & 158 & $100 \%$
\end{tabular}

Demographics

Male

Female

Age (median)

Mechanism of trauma

Inversion

Eversion

Unknown

Activities

Walking

Sports

Traffic accident

Other

OAR

Rule 1 (4 steps walking test)

Rule 2 (lateral malleolus)

Rule 3 (medial malleolus)

Rule 4 (fifth metatarsal bone)

Rule 5 (navicular)

PoCUS fracture sonographer

No fracture

Fracture

Distal tibia

Distal fibula

Fifth metatarsal bone

Distal fibula and tibia

Other

PoCUS fracture expert

No fracture

Fracture

Distal tibia

Distal fibula

Fifth metatarsal bone

Distal fibula and tibia

Other

Xray fracture

No fracture

Fracture

Distal tibia

Distal fibula

Fifth metatarsal bone

Distal fibula and tibia

Other $^{\text {a }}$
61

97

$28(21-46)$

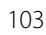

10

45

79

54

16

3

117

111

69

54

44 
Table 3 Clinically relevant fractures seen on radiography compared to point-of-care bedside ultrasound and sensitivity, specificity, positive predictive value (PPV) and negative predictive value (NPV) of the sonographers and expert in significant fractures

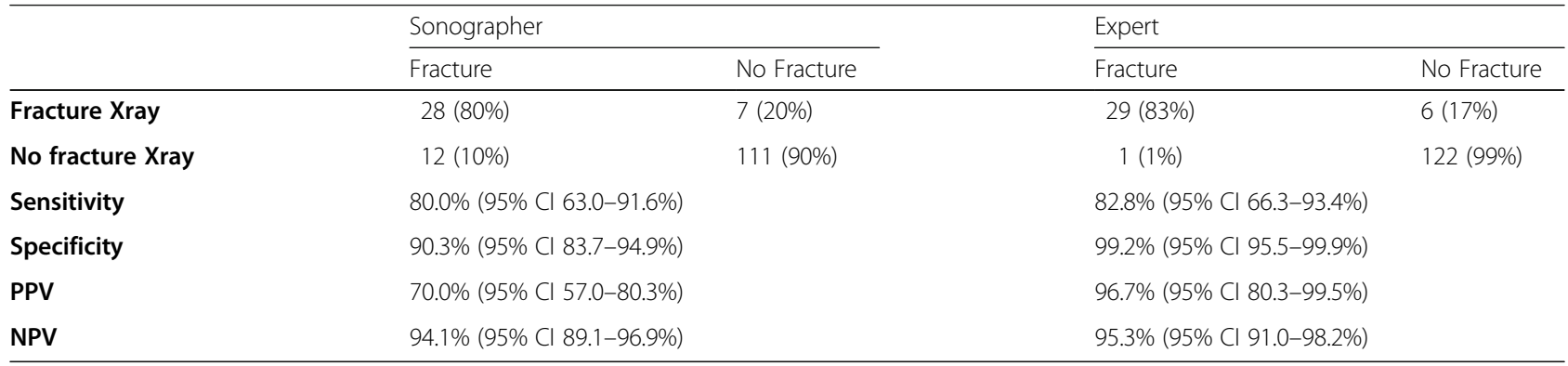

CI 95.5 to $99.9 \%)$, positive predictive value $96.7 \%(95 \%$ CI 80.3 to $99.5 \%)$ and the negative predictive value 95.3\% (95\% CI 91.0 to $98.2 \%$ ), see Table 3.

There were no adverse events from performing PoCUS or the reference standard, radiography.

\section{Discussion}

This prospective cohort study, which is the most relatable to usual clinical practice so far, found PoCUS to have a high specificity and negative predictive value in diagnosing significant fractures of the ankle and foot [9].

PoCUS performed in consecutive patients by all sonographers, with different levels of experience, results in a sensitivity of $80.0 \%$ and specificity of $90.3 \%$. This is less compared to previous studies $[8,10]$. However, these studies were not blinded and used only 1-5 sonographers, which is not realistic in usual clinical practice. In common clinical practice, sonography will be performed by both EP's as well as residents, with a difference in experience in PoCUS. This usual clinical practice is well reflected in our study, where the experience amongst the 23 sonographers varied in PoCUS for different uses and specifically this indication, as well as the exposure to foot and ankle trauma in our tertiary centre.

For this study, sonographers were blinded for the history and, as far as possible, abnormalities shown in the physical examination and positive rules of the OAR. This was the most valid way to study sensitivity and specificity of PoCUS only, without bias. In usual clinical practice, PoCUS would be executed by the same person taking the history and performing the physical examination. Thus, combining clinical knowledge with the abnormalities seen with PoCUS may very well further improve the diagnostic value of this test.

The results of the expert in our study show that more experience with PoCUS for this indication and frequent exposure will improve diagnostic value. Also, PoCUS is a dynamic examination, and when assessing an exam performed by someone else, interpretation might be different, which explains the missed fractures of the expert. However, when compared to previous studies, the expert had comparable diagnostic value, even whilst being blinded for the history and clinical examination $[8,10]$.

It appears that, using PoCUS in acute foot and ankle trauma, albeit a high negative predictive value, there is a risk of missing fractures and only a minimal risk for false positive fractures. When instructing the patient, who has a negative PoCUS exam on presentation, to return for additional assessment if complaints persist or aggravate, missed fractures can be intercepted and treated accordingly.

This sensitivity corresponds to the 12 false positive fractures $(10 \%)$ diagnosed by the sonographers. False

Table 4 Demographics of the nine missed fractures

\begin{tabular}{|c|c|c|c|c|c|c|c|}
\hline Age & gender & Trauma mechanism & Activity & Fracture & Sonographer no & Sonographer seen & Expert seen \\
\hline 24 & male & Inversion & sports & MT V & 1 & no & yes \\
\hline 18 & female & Inversion & walking & MT V & 2 & no & yes \\
\hline 23 & female & Unknown & walking & MT V (Jones) & 4 & no & no \\
\hline 57 & female & Unknown & walking & distal fibula & 3 & no & no \\
\hline 55 & female & Unknown & traffic accident & MT V & 3 & no & no \\
\hline 19 & female & Eversion & traffic accident & distal tibia + malleolus tertius & 7 & no & yes \\
\hline 19 & female & Unknown & traffic accident & distal fibula & 5 & no & no \\
\hline 23 & female & Eversion & walking & distal fibula & 6 & yes & no \\
\hline 19 & male & Unknown & sports & distal fibula & 8 & yes & no \\
\hline
\end{tabular}


positive fractures would result in treating the patient avoidably as a fracture, with a backslap, which is also an accepted treatment for sprain. In this case usual clinical practice would be to assess the patient clinically within a week, which could prevent longer immobilisation.

In this study an overall $76 \%$ absence of fractures, confirmed with radiography, was comparable to known results of the OAR [4]. If patients were assessed with PoCUS by a sonographer in combination with the OAR alone, radiographic imaging could have been prevented in $80 \%$, the amount of rightly diagnosed non-fractures. The high negative predictive value of the test supports these findings. This is in accordance with previous studies, which also show a reduction of radiological assessment of approximately $80 \%[2,5]$.

Patients with negative OAR and PoCUS, could have been treated for sprain or avulsion fracture in the prehospital setting without presentation to the ED. With the current increase in crowding in the ED, PoCUS for pre-hospital or general practitioner triage could be beneficial. This accounts for outpatient clinics and the developing world (where radiology is expensive), as recommended by The World Health Organization [1]. Besides this, portable handheld PoCUS is already available and might be implemented in future hospital settings, making quick bedside diagnostics accessible.

\section{Limitations}

This study was conducted in an academic tertiary hospital, which may result in bias especially for results in primary or secondary care. There were more ineligible patients than expected, due to missing data, nonassessable videos or because blinding was not guaranteed (thus when patients were included by the expert sonographer). The missing data caused a certain degree of bias. During the study period the ultrasound machine un-expectantly broke down resulting in a temporary pause in inclusion and images that could not be saved correctly or were lost in the process. Total blinding could not always be assured because of visible hematoma and swelling in foot and ankle trauma, however, this reflects common practice. In young patients under 20 years, the epiphyseal plate could still be present, in which case the hypoechoic appearance of the epiphyseal cartilage might appear as a cortical disruption when PoCUS is being performed by less experienced sonographers. However, there is a small probability of a still existing epiphyseal plate in the number of patients $\geq 17$ years and under 20 years in this study.

\section{Conclusion}

PoCUS combined with the OAR has a good diagnostic value in usual clinical practice in the assessment of suspected ankle and fifth metatarsal bone fractures compared to radiographic imaging. In this study, we show that more experience with PoCUS and frequent exposure will further improve diagnostic value. Implementing PoCUS for the evaluation of ankle and fifth metatarsal bone trauma in the ED can possibly reduce the use of radiography and minimize the exposure to radiation, time, costs and burdening of the patient and ED.

\section{Abbreviations \\ ED: Emergency Department; EP: Emergency physician; OAR: Ottawa Ankle Rules; PoCUS: Point-of-care bedside ultrasound; STARD: Standards for Reporting Diagnostic Accuracy; Cl: Confidence interval}

\section{Acknowledgements}

Bram Smit made contributions to the acquisition and the analysis of the data.

\section{Authors' contributions}

Aniek Crombach made substantial contributions to the conception and design of the work and the acquisition, analysis, and interpretation of data, drafted the work or substantively revised it, has approved the submitted version (and any substantially modified version that involves the author's contribution to the study and to has agreed both to be personally accountable for the author's own contributions and to ensure that questions related to the accuracy or integrity of any part of the work, even ones in which the author was not personally involved, are appropriately investigated, resolved, and the resolution documented in the literature. Nasim Azizi made substantial contributions to the conception and the acquisition of the work, has approved the submitted version and agreed both to be personally accountable for the author's own contributions and to ensure that questions related to the accuracy or integrity of any part of the work, even ones in which the author was not personally involved, are appropriately investigated, resolved, and the resolution documented in the literature. Heleen Lameijer made substantial contributions to the analysis of data, substantively revised the work, and has approved the submitted version that involves the author's contribution to the study and to has agreed both to be personally accountable for the author's own contributions and to ensure that questions related to the accuracy or integrity of any part of the work, even ones in which the author was not personally involved, are appropriately investigated, resolved, and the resolution documented in the literature. Mostafa El

Moumni made substantial contributions to the conception and design of the work, has approved the submitted version and agreed both to be personally accountable for the author's own contributions and to ensure that questions related to the accuracy or integrity of any part of the work, even ones in which the author was not personally involved, are appropriately investigated, resolved, and the resolution documented in the literature. Jan C. ter Maaten made substantial contributions to the conception and design of the work and interpretation of data, has approved the submitted version and agreed both to be personally accountable for the author's own contributions and to ensure that questions related to the accuracy or integrity of any part of the work, even ones in which the author was not personally involved, are appropriately investigated, resolved, and the resolution documented in the literature. The author(s) read and approved the final manuscript.

\section{Funding}

This study was financially supported by the Spoedeisende Geneeskunde Onderzoeksfonds (Netherlands Emergency Medicine Research Fund).

\section{Availability of data and materials}

Dataset will not be online available because individual privacy could be compromised. The dataset is available and archived as electronic file in the emergency department of the UMCG.

\section{Ethics approval and consent to participate}

Approval by the local ethics committee was obtained and patients informed consent documented, number M15.176601. 


\section{Consent for publication}

Patients informed consent was documented for each patient.

\section{Competing interests}

None for all authors.

\section{Author details}

'Department of Emergency Medicine, University Medical Centre Groningen University of Groningen, Hanzeplein 1, 9700 RB Groningen, the Netherlands. ${ }^{2}$ Department of Emergency Medicine, Medical Centre Leeuwarden, Leeuwarden, the Netherlands. ${ }^{3}$ Department of Surgery, University Medical Centre Groningen, University of Groningen, Groningen, the Netherlands. ${ }^{4}$ Department of Internal Medicine, University Medical Centre Groningen, University of Groningen, Groningen, the Netherlands.

Received: 14 November 2019 Accepted: 23 April 2020

Published online: 07 May 2020

\section{References}

1. Hedellin H, Goksör LA, Karlsson J, Stjernström S. Ultrasound-assisted triage of ankle trauma can decrease the need for radiographic imaging. Am J Emerg Med. 2013;31:1686-9.

2. Jonckheer P, Willems T, Ridder RD, Paulus D, Henningsen KH, Miguel LS, et al. Evaluating fracture risk in acute ankle sprains: any news since the Ottawa ankle rules? A systematic review. Eur I Gen Pract. 2016;22(1):31-41.

3. Stiell IG, Bennet C. Implementation of clinical decision rules in the emergency department. Acad Emerg Med. 2007;14(11):955-9.

4. Bachmann LM, Kolb E, Koller MT, Steurer J, ter Riet G. Accuracy of Ottawa ankle rules to exclude fractures of the ankle and mid-foot: systematic review. BMJ. 2003;326(7386):417.

5. Canagasabey M, Callaghan MJ, Carley S. The Sonographic Ottawa foot and ankle rules study (the SOFAR study). Emerg Med J. 2011;28:838-40.

6. Bossuyt PM, Reitsma JB, Bruns DE, Gatsonis CA. STARD 2015: an updated list of essential items for reporting diagnostic accuracy studies. Radiology. 2015; 277(3):826-32.

7. Fiesseler F, Szucs P, Kec R, Richman PB. Can nurses appropriately interpret the Ottawa ankle rule? Am J Emerg Med. 2004;22(3):145-8.

8. Ekinci S, Polat O, Günalp M, Demirkan A, Koca A. The accuracy of ultrasound evaluation in foot and ankle trauma. Am J Emerg Med. 2013;31(1):1551-5.

9. Chartier LB, Bosco L, Lapointe-Shaw L, Chenkin J. Use of point-of-care ultrasound in long bone fractures: a systematic review and meta-analysis. Can J Emerg Med. 2017;19(2):131-42.

10. Atilla OD, Yesilaras M, Kllic TY, Tur FC, Reisoglu A, Sever M, et al. The accuracy of bedside ultrasonography as a diagnostic tool for fractures in the ankle and foot. Acad Emerg Med. 2014;21:1058-61.

11. The Ultrasound Leadership Academy. Available from: http://www. ultrasoundleadershipacademy.com/ULA/one-page/index.html. Accessed 4 Sept 2019.

12. Jones SR, Carley S, Harrison M. An introduction to power and sample size estimation. Emerg Med J. 2003;20(5):453-8.

\section{Publisher's Note}

Springer Nature remains neutral with regard to jurisdictional claims in published maps and institutional affiliations. 